\title{
HEALTHCARE DELIVERY
}

\section{Taking kangaroo mother care forward in South Africa: The role of district clinical specialist teams}

\author{
U Feucht, E van Rooyen, R Skhosana, A-M Bergh
}

Ute Feucht is the paediatrician on the Tshwane District Clinical Specialist Team, Tshwane District Health Services, South Africa, and an adjunct professor in the Department of Paediatrics, Faculty of Health Sciences, University of Pretoria and Kalafong Hospital, Pretoria, South Africa. Elise van Rooyen is a medical officer in the Department of Paediatrics, Kalafong Hospital, and a lecturer in the Department of Paediatrics, University of Pretoria. Rinah Skhosana is the paediatric nurse on the Tshwane District Clinical Specialist Team. Anne-Marie Bergh is a senior researcher at the MRC Unit for Maternal and Infant Health Care Strategies, Faculty of Health Sciences, University of Pretoria.

Corresponding author: U Feucht (ute.feucht@up.ac.za)

The global agenda for improved neonatal care includes the scale-up of kangaroo mother care (KMC) services. The establishment of district clinical specialist teams (DCSTs) in South Africa (SA) provides an excellent opportunity to enhance neonatal care at district level and ensure translation of policies, including the requirement for KMC implementation, into everyday clinical practice. Tshwane District in Gauteng Province, SA, has been experiencing an increasing strain on obstetric and neonatal services at central, tertiary and regional hospitals in recent years as a result of growing population numbers and rapid up-referral of patients, with limited down-referral of low-risk patients to district-level services. We describe a successful multidisciplinary quality improvement initiative under the leadership of the Tshwane DCST, in conjunction with experienced local KMC implementers, aimed at expanding the district's KMC services. The project subsequently served as a platform for improvement of other areas of neonatal care by means of a systematic approach. 


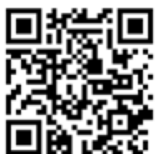

Low birth weight (LBW) and prematurity are significant contributors to neonatal mortality and have become major barriers in reaching child mortality targets. ${ }^{[1]}$ Kangaroo mother care (KMC) is a high-impact, lowtech, cost-effective intervention to reduce neonatal mortality and morbidity. ${ }^{[2]}$ It has proven advantages in terms of infant feeding and weight gain and reduced infection risks, as well as improved maternal physical and psychological health, with an accompanying reduction in health institution utilisation. ${ }^{[3-7]}$ The KMC components include securing infants skin-to-skin to their mothers' chests in an upright position by means of a cloth or wrap, exclusive breastfeeding, early hospital discharge and adequate support of the mother-infant pair by health workers and family members. ${ }^{[8]}$

The current global implementation and research agenda for improved newborn care includes accelerated KMC scale-up. ${ }^{[2,9]}$ KMC has also become part of South African (SA) health policy through initiatives such as the Tshwane Declaration ${ }^{[10]}$ and the Campaign on Accelerated Reduction of Maternal and Child Mortality in Africa. ${ }^{[11]}$ The primary healthcare re-engineering process currently under way in SA is an excellent opportunity to enhance neonatal care and ensure translation of these policies into practice. ${ }^{[12]}$ The district clinical specialist teams (DCSTs), comprising senior medical and nursing personnel in the fields of primary, maternal, paediatric and emergency care, form one of the pillars of this health system improvement initiative. ${ }^{[13,14]}$ Their roles include training, supportive supervision, clinical governance and helping to establish the necessary linkages between hospital-based neonatal units, primary healthcare facilities, district-based health programme staff and communitybased organisations, all of which can potentially greatly enhance the neonatal care continuum. DCSTs are not facility based and are therefore not constrained by systems boundaries between the different levels of healthcare, which enables the members to move between the various health facilities in order to facilitate care networks, with an anticipated impact at health systems level.

This article describes a quality improvement process undertaken in the Tshwane District, Gauteng Province, in which strengthening of KMC was used as an entry point for the improvement of neonatal care under the supervision of the DCST. The district, which covers the northern part of Gauteng and has an urban and rural population of about three million people, has undergone major changes in the past 15 years with new boundary demarcations adding two district hospitals to the health facilities requiring oversight. ${ }^{[15]}$ Currently there are two central, one tertiary, one regional and four district hospitals. The Tshwane DCST was established in 2012 and has a full complement of staff that includes three specialists, two medical officers and three professional nurses with advanced training.

\section{Making $\mathrm{KMC}$ work in practice}

Initial KMC training and implementation initiatives in Gauteng ${ }^{[16]}$ had limited impact at scale as various factors hindered full KMC implementation in all hospitals. By 2013 Tshwane District had 45 KMC beds at tertiary institutions, 5 beds at the regional hospital and only $20 \mathrm{KMC}$ beds for down-referrals at district hospital level. The district's expanding population, with a resultant increase in deliveries, was placing a strain on obstetric and neonatal services at the large hospitals. The growing neonatal bed shortages were compounded by rapid up-referral of patients from district hospitals to higher levels of care, with very few patients reversely down-referred to district hospitals. The DCST, in conjunction with experienced local KMC implementers, identified this gap and jointly embarked on a quality improvement initiative from 2013 to 2015 to facilitate KMC scale-up. The activities included three workshops and one walk-through visit to each hospital to provide support and assess the level of facility-based KMC implementation.

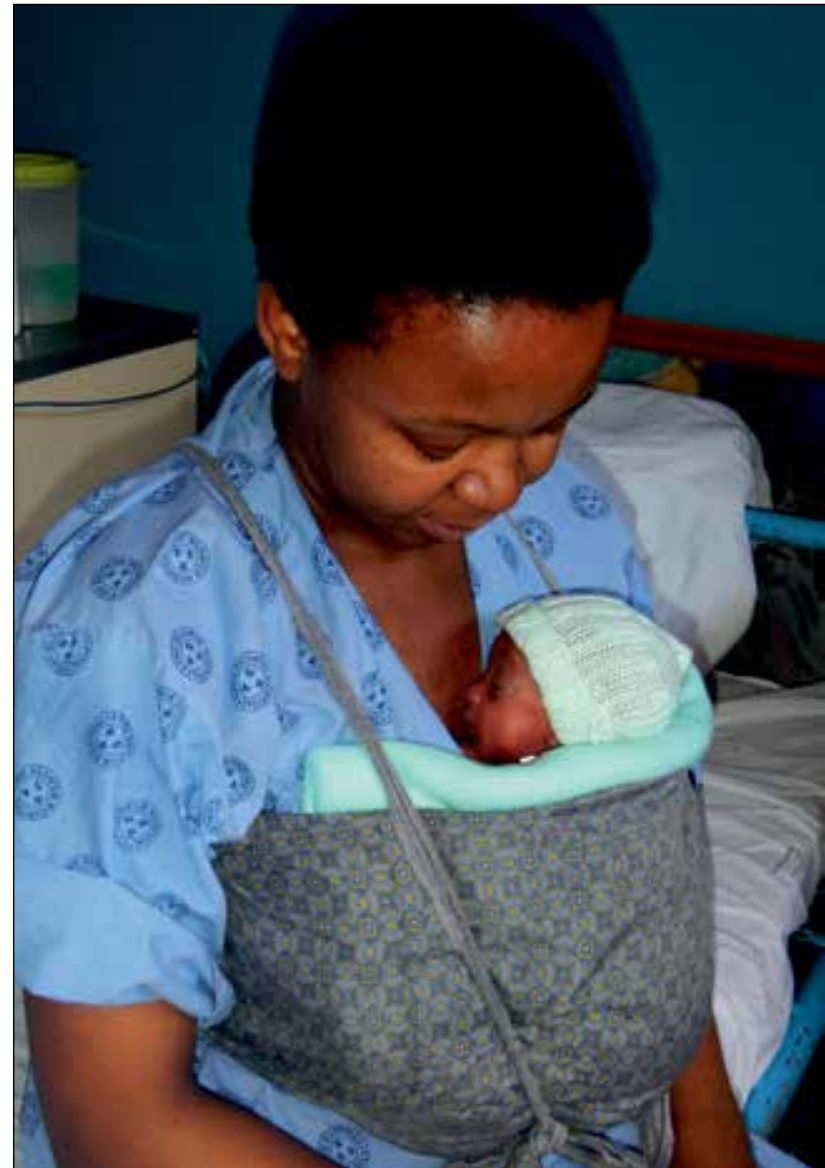

Training workshops with a multidisciplinary team from each hospital were held in November 2013 and April 2014 and were attended by doctors, nurses, dieticians and other allied health workers. Additional attendees were members of the health system support structures, namely the Tshwane District Maternal, Newborn, Child and Women's Health (MNCWH) and Nutrition subdirectorate. The Tshwane DCST members provided workshop support and were upskilled simultaneously. Each workshop comprised two main components: feedback on each hospital's KMC implementation process (including achievements and challenges), and training sessions. Struggling hospitals were encouraged to conduct benchmark visits to hospitals with well-established KMC units and to identify local champions to initiate KMC implementation with the support of the DCST.

In July 2014, every hospital was visited by a team consisting of the DCST, experienced local KMC implementers and other members from the Tshwane District office. The walk-through visits also provided an in-service training opportunity for capacity building through supportive supervision. A standard progress monitoring tool was used for evaluating KMC implementation in each hospital. ${ }^{[16,17]} \mathrm{A}$ follow-up workshop was then held in May 2015 to ensure continued feedback from facilities on their KMC implementation progress and to discuss further topics related to the improvement of $\mathrm{KMC}$ practice and services.

\section{Lessons learned: The rolle of DCSTs in KMC implementation}

The project-type approach to advancing a specific aspect of neonatal care - in this case KMC implementation - was an excellent vehicle for implementing health systems improvements. Ongoing monitoring and evaluation of neonatal healthcare was already in place, but a focused intervention was required to strengthen KMC services at all hospitals through a team-orientated approach, with subsequent expansion 


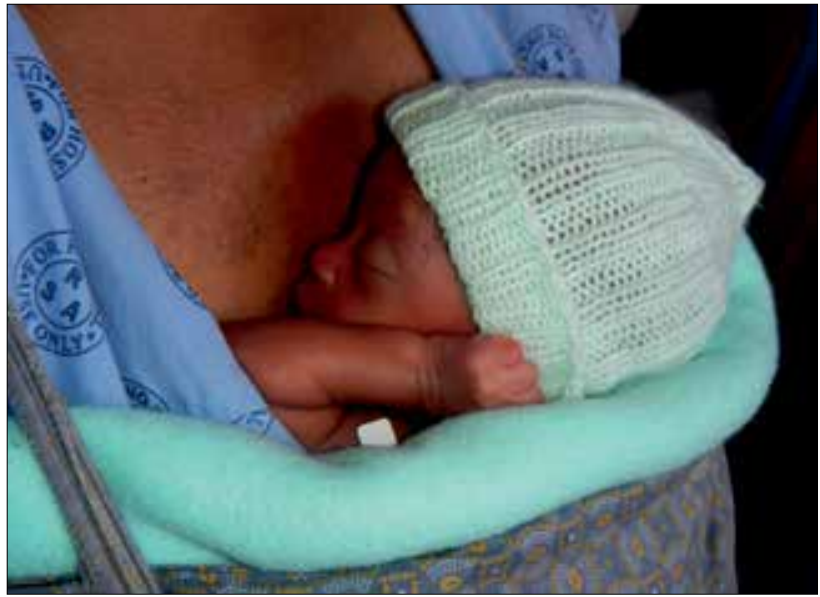

to other neonatal care issues. This quality improvement initiative was introduced without additional funding, which demonstrates how resources can be mobilised to effect change. The process was furthermore enabled by national and provincial priority setting, with existing policies and guidelines strengthening the DCST's ability to encourage all hospitals to participate, with the necessary support of the hospital management.

Empowerment of staff in the participating hospitals was achieved through workshops and on-site visits, with accompanying mentoring through a non-hierarchical approach. The equal empowering relationships helped promote ownership at hospital and district level, with mutual benchmarking visits leading to a high degree of transfer of learning.

Insights that emerged from the process included:

- The importance of the non-separation of mother-infant pairs to encourage bonding and give babies a 'voice' through their mothers

- The empowerment of mothers to take responsibility for the care for their LBW and preterm babies under medical and nursing supervision, with a focus on 'special-needs groups' with social or medical risk factors

- The need for onsite protocols and guidelines

- The integration of KMC with other maternal and child health programmes at facility level (e.g. family planning, HIV prevention and immunisation services), with the KMC environment providing the time and space for services to be delivered to these high-risk populations

- The need to provide KMC wraps to ensure continued KMC practice after discharge.

Successful KMC implementation requires partnerships between neonatal and obstetric care workers, family physicians and primary healthcare nurses, illustrating the importance of a multidisciplinary team approach to effect change, with team members from different disciplines complementing each other. Collaboration between tertiary and district hospitals was additionally enabled. The workshops provided an egalitarian platform for dealing with weaknesses in the system in a relatively formal way, with discussions around particular index patients leading to further discussion on systems improvements.

The partnerships stretched beyond the DCST and included MNCWH and Nutrition staff members, as well as other clinical and management experts working at health institutions within the district boundaries. This provided the potential for the combination of clinical expertise, mentoring and health systems improvements. Within any given DCST the required expertise to address particular clinical issues may not be available, but project implementation may benefit greatly from the inclusion of local experts. KMC units in the Tshwane hospitals additionally strengthened their collaboration with the in-facility allied health workers.
The permanent presence of the DCST in the district ensures continuous supportive supervision, thereby enabling accountability, mentoring and follow-up with encouragement of the onsite staff members beyond a once-off project. Relationships of trust and local ownership were identified as the basic characteristics of supportive supervision in a context in which home-grown solutions can be promoted successfully. Local initiative with the development of protocols and guidelines was supported, but the DCST also had sufficient authority to implement and monitor the use of protocols and guidelines in hospitals showing slow progress.

The chosen team approach, together with appropriate supportive supervision, had the potential ability to influence or effect change. Improvements included appropriate medical care for late preterm infants, correct use of a breastmilk fortifier, and improved breastfeeding by mothers after hospital discharge. Infrastructural constraints were highlighted with an emphasis on the provision of a thermoneutral environment in all areas designated for neonatal care. While the project was being implemented the number of KMC beds in the district hospitals increased from 20 to 39, thereby almost doubling the KMC capacity. Additionally, improved cross-referrals were observed between hospitals in terms of specialised neonatal follow-up services. Staff morale and enthusiasm visibly improved in the period during which the workshops and support visits were held, and the DCST members had the authority to work with clinicians and facility management in order to address local KMC barriers that appeared insurmountable at times.

\section{Conclusion}

Mortality and morbidity in preterm and LBW infants can be reduced through healthcare interventions for the mother-infant pair before and during pregnancy and after birth ${ }^{[18]}$ The service provision of the relevant healthcare focus areas needs to be institutionalised and not individually driven, in order to reduce fluctuation in service delivery with staff mobility. At the same time the maintenance of the neonatal care continuum relies on teamwork between the different care providers. The KMC implementation project described here was successful in improving KMC services in the Tshwane district, and it served as a platform from which to improve other areas of neonatal care by using a systematic approach.

Acknowledgements. The support of the members of the Tshwane DCST and MNCWH and Nutrition subdirectorate is acknowledged with thanks. Managers and clinical staff at the hospitals are thanked for their collaboration.

1. Howson C, Kinney M, McDougall L, Lawn J, on behalf of the Born Too Soon Preterm Birth Action Group. Born too soon: Preterm birth matters. Reprod Health 2013;10(Suppl):S1. http://www.reproductive-health-journal.com/ content/10/S1/S1 (accessed 29 September 2015).

2. World Health Organization. Every Newborn: An Action Plan to End Preventable Deaths. Geneva: WHO, 2014.

3. Conde-Agudelo A, Diaz-Rossello J. Kangaroo mother care to reduce morbidity and mortality in low birthweight Conde-Agudelo A, Diaz-Rossello J. Kangaroo mother care to reduce morbidity and mortality in low birthweight
infants. Cochrane Database Syst Rev 2011, Issue 3. Art. No.: CD002771. [http://dx.doi.org/10.1002/14651858. infants. Cochrane

4. Lawn JE, Mwansa-Kambafwile J, Horta BL, Barros FC, Cousens S. Kangaroo mother care to prevent neonatal deaths due to preterm birth complications. Int J Epidemiol. 2010;39(Suppl 1):i144-1154. [http://dx.doi.org/10.1093/ije/dyq031] 5. Ludington-Hoe SM, Morgan K, Abouelfettoh A. A clinical guideline for implementation of kangaroo care with premature infants of 30 or more weeks' postmenstrual age. Adv Neonatal Care 2008;8(3 Suppl):S3-S23. [http://dx.doi. org/10.1097/01.ANC.0000324330.25734.b6]

6. Affonso D, Bosque E, Wahlberg V, Brady J. Reconciliation and healing for mothers through skin-to-skin contact provided in an American tertiary level intensive care nursery. Neonatal Netw 1993;12(3):25-32.

7. De Alencar A, Arraes L, de Albuquerque E, Alves J.Effect of kangaroo mother care on postpartum depression.J Trop Pediatr 2009;55(1):36-38. [http://dx.doi.org/10.1093/tropej/fmn083]

8. Ruiz JG, Charpak N, et al. Evidence-based Clinical Practice Guidelines for an Optimal Use of the Kangaroo Mother Method in Preterm and/or Low Birthweight Infants at Birth. Bogotá: Fundación Canguro and Department of Method in Preterm and/or Low Birthweight Infants at Birth. Bogotá: Fundación Canguro and Dex
Clinical Epidemiology and Biostatistics, School of Medicine, Pontificia Universidad Javeriana, 2007.

9. Enical Epidemiology and Biostatistics, School of Medicine, Pontificia Universidad Javeriana, 2007 . . Engmann C, Wall S, Darmstadt G, Valsangkar B, Claeson M, on behalf of the participants of the Istanbul KMC
Acceleration Meeting. Consensus on kangaroo mother care acceleration. Lancet 2013;382(9907):e26-e27. [http://

dx.doi.org/10.1016/S0140-6736(13)62293-X]
0. The Tshwane declaration of support for breastfeeding in South Africa. S Afr J Clin Nutr 2011;24(4):214.

11. Department of Health, Republic of South Africa. South Africas national strategic plan for a campaign on accelerated reduction of maternal and child mortality in Africa (CARMMA) 2012. http://www.hst.org.za/sites/default/files/ carmma.pdf (accessed 30 June 2014).

12. Naidoo S. The South African national health insurance: A revolution in health-care delivery! J Public Health 2012;34(1):149-510. [http://dx.doi.org/10.1093/pubmed/fds008]

13. Ministerial Task Team. District Clinical Specialist Teams in South Africa. Ministerial Task Team Report to the Honourable Minister of Health, Dr Aaron Motsoaledi. 2012. http://www.rmchsa.org/wp-content/resources/ resources_by_type/DistrictLevelResources/MinisterialTTReport_DCSTInSouthAfrica.pdf (accessed 13 February 2013). 
14. Connell L. A Clinical Governance Handbook for District Clinical Specialist Teams. Durban: Health Systems Trust, 2014.

15. Ganief A, Thorpe J. City of Tshwane General and Regional Overview. Cape Town: Parliament of the Republic of South Africa, 2013. http://www.parliament.gov:za/content/Tshwane_General_and_Regions_Report_2013.pdf South Africa, 2013. http//m

16. Bergh $A-M$, van Rooyen E, Pattinson RC. 'On-site’ versus 'off-site facilitation: A randomised trial of outreach strategies 6. Bergh A-M, van Rooyen E, Pattinson RC. On-site versus off-site facilitation: A randomised trial of outreach strateg
for scaling up kangaroo mother care. Hum Resour Health 2008; 6 :13. [http://dx.doi.org/10.1186/1478-4491-6-13]
17. Bergh A-M, Arsalo I, Malan A, Pattinson R, Patrick M, Phillips N. Measuring implementation progress in kangaroo mother care. Acta Paediatr 2005;94(8):1102-1108. [http://dx.doi org/10.1080/08035250510028380]

18. World Health Organization. WHO Recommendations on Interventions to Improve Preterm Birth Outcomes. Geneva: WHO, 2015

Accepted 27 October 2015.

\section{W SMAMA Delease}

\section{Freezing healthcare posts "counter-productive," say doctors}

The freezing of critical physician and nursing posts in at least five provinces is "dangerously short-sighted" and will hurt vulnerable patient populations, boost billion rand litigation costs and aggravate working conditions, the SA Medical Association warned yesterday.

Introduced as austerity measures in stark contrast to the mushrooming of middle and senior management placements in recent years, the freezing of these critical service delivery posts this December and January comes exactly when most doctors and nurses are seeking them. Chairman of SAMA, Dr Mzukisi Grootboom said this would result in lost opportunities and chronic staffing shortages for 2016 - with multiple negative knock-on effects. "Although mention is made of a process whereby critical posts can be unfrozen and advertised, the reality is that this is a long bureaucratic procedure which may require approval from the premier's office. Over the last few years we have seen a mushrooming of middle and senior management positions in the various provincial departments of health at district and provincial level, with a net decrease in service level posts at institution level," Grootboom added. The provinces that have frozen the healthcare staff posts are; the North West, Eastern Cape, KwaZulu-Natal, Mpumalanga and Free State.

The SAMA chairperson said many provincial departments of health had received qualified audits by the auditor general, indicating irregular and fruitless expenditure as the primary cause for not achieving a clean audit. Many of their problems were caused by dismal supply chain management, a major contributor to their financial woes.

The SAMA believed that the freezing of critical posts would have a negative impact, not only on patient care, but on working conditions and adverse events in health care. Litigation against departments of health have shown an alarming upward spiralling trend over the last few years with national health minister, Dr Aaron Motsoaledi, calling a summit to deal with this in March and the biggest private sector risk underwriter, the Medical Protection Society, (MPS), appealing for legal reforms at a similar seminar held in November. Grootboom said this litigation trend was set to continue unless the root causes were effectively dealt with.

\section{Training more doctors pointless if you can't employ them}

In addition, the recent drive by the National Health Department to increase the number of medical graduates seemed pointless "if conditions on the floor prevent recruitment and retention of staff." Many qualified doctors and specialists were forced to seek employment in the well-resourced private sector or emigrate because of the lack of available posts in the public sector. In addition, the training of medical students was often compromised as the burden of service delivery commitments prevented the more experienced doctors from being available to teach and train them.

Grootboom said that when austerity measures were introduced the first posts to be targeted were the medical ones, because there were always medical vacancies in the public sector due to the mobility of doctors. All public healthcare institutions had workloads which needed to be serviced by a defined staffing level. "The freezing of medical officer, specialist and registrar posts is very short sighted and will only serve to further compromise the quality of care being rendered by the public healthcare service to the most vulnerable populations in South Africa," he concluded.

Contact:

Head of PR \& Communications

Dr Simonia Magardie

Spokesperson

Chairperson: SAMA

Dr Mzukisi Grootboom 